\title{
ESTRATEGIA DIDÁCTICA PARA EL APRENDIZAJE CONCEPTUAL DE LAS DESIGUALDADES A TRAVÉS DE LAS REPRESENTACIONES SEMIÓTICAS
}

\section{DIDACTIC STRATEGY FOR CONCEPTUAL LEARNING OF INEQUALITIES THROUGH SEMIOTIC REPRESENTATIONS}

\author{
Fabio Durán Salas1
}

\section{RESUMEN}

Esta investigación propone una estrategia didáctica para el aprendizaje conceptual de las desigualdades desarrollada con estudiantes entre los 15 y 16 años para dinamizar la interpretación, comprensión y solución de situaciones tanto en contextos matemáticos y como en contextos cotidianos, teniendo referente metodológico las representaciones conceptuales, los tratamientos y las conversiones semióticas como las tres fases del desarrollo conceptual de las desigualdades. La estrategia

$1 \quad$ Candidato a doctor en ciencias de la educación de la Universidad de San Buenaventura (Colombia). Integrante del grupo de investigación ESINED (USB-Medellín). Correo electrónico: fabio.duran@aspaen.edu.co. Orcid: https://orcid.org/0000-0003-1153-5441 se desarrolló en sesiones de aula en las que se trabajaron múltiples representaciones de las desigualdades para resolver inecuaciones, determinar el dominio y codominio de funciones logarítmicas, racionales e irracionales y conversiones semánticas de un perfil lipídico.

PALABRAS CLAVE: Estrategia didáctica, aprendizaje conceptual, representaciones semióticas, tratamiento semiótico y conversión semiótica

\section{ABSTRACT}

This research proposes a didactic strategy for the conceptual learning of inequalities developed with students between 15 and 16 years of age to dynamize the interpretation, 
understanding, and solution of situations both in mathematical contexts and in daily contexts, having as methodological reference the conceptual representations, treatments, and semiotic conversions as the three phases of the conceptual development of inequalities. The strategy was developed in classroom sessions in which multiple representations of inequalities were worked on to solve inequalities, determine the domain and codomain of logarithmic functions, rational functions, irrational functions, and semantic conversions of a lipid profile.

Keywords: didactic strategy, conceptual learning, semiotic representations, semiotic treatment, and semiotic conversion.

\section{INTRODUCCIÓN}

Múltiples investigaciones muestran que el aprendizaje conceptual es una potente herramienta que ayuda a los estudiantes a organizar la información de manera lógica y coherente, movilizando significativamente los conceptos antiguos con los nuevos para aplicarlos en diversos contextos y solucionar problemas cotidianos (Geary et al., 2017). De ahí que, los procesos de conceptualización de la actividad matemática necesariamente deben pasar por registros de representación semiótica para ser comunicados y aprendidos (D'Amore, 2003). Efectivamente, los registros de representación semiótica tienen dos funciones, (i) le permite al aprendiz entrar en contacto con el objeto matemático (lori, 2016); (ii) pone al objeto matemático en un nivel de aprendizaje para que los procesos de tratamiento y conversión de las representaciones semióticas le proporcionen sentido y significado a los conceptos (Fandiño, 2010).

Sin embargo, el aprendizaje conceptual de las desigualdades matemáticas enfrenta múltiples desafíos didácticos por la complejidad semiótica en su proceso de aprendizaje y por la relevancia que tiene en diversas disciplinas del conocimiento, así mismo, "la experiencia con la enseñanza de las desigualdades, ha permitido observar que muchos de los errores que cometen los estudiantes vuelven a ocurrir año tras año" (Blanco \& Garrote, 2007, p. 221)En efecto, numerosas investigaciones han estudiado diferentes obstáculos que presentan los estudiantes para desarrollar tareas específicas con las desigualdades (Almog \& Ilany, 2012; Balomenou et al., 2017; Blanco \& Garrote, 2007; Hattikudur \& Alibali, 2010; Sangwin, 2015; Sierpinska et al., 2011; P. Tsamir \& Almog, 2001; Pessia Tsamir \& Bazzini, 2004). Sin embargo, son casi inexistentes las investigaciones que proponen estrategias que puedan ser aplicadas por profesores en el salón de clase para que los estudiantes puedan adquirir y desarrollar habilidades con desigualdades. Dentro del marco de las dificultades que exteriorizan los estudiantes se sintetizan dos categorías; la primera, precisa que la enseñanza de las desigualdades se reduce a tareas mecánicas y no de apropiación del contenido semántico de cada propiedad de las desigualdades. Por otro lado, los estudiantes establecen relaciones limitadas entre los diferentes sistemas de representación semiótica de las desigualdades. La segunda, se analizan diferentes errores que generalmente incurre los estudiantes al aprender desigualdades, a saber: (i) no cambiar el signo cuando se multiplica o divide por un número negativo; (ii) al resolver desigualdades no relacionan los resultados con sus representaciones gráficas y (iii) la enseñanza y el aprendizaje de las inecuaciones se centra en el formalismo lógico de sus propiedades. Por lo anterior se hace necesario, centrar la atención en la introducción del concepto de desigualdad en las actividades de aula, ya que de lo contrario, cualquier aprendizaje memorístico o mecánico puede conllevar a los estudiantes a cometer errores con frecuencia (Blanco \& Garrote, 2007)

La construcción cognitiva para el aprendizaje de las desigualdades está estrechamente 
relacionada con la capacidad de usar varios registros de representación semiótica para su entendimiento, compresión y sentido (Balomenou et al., 2017. Así mismo, la aplicabilidad conceptual se extiende a múltiples contextos de la actividad humana relacionadas con el lenguaje y el entorno natural; por ejemplo, "Antonio tiene entre 25 y 30 años", "hoy la temperatura está entre $20^{\circ} \mathrm{C}$ y $25^{\circ} \mathrm{C}$ "; "se demora entre media hora y una hora llegar hasta allí"; "para el 2022 el PIB estará sobre el 2.2", y en pruebas de laboratorio como un perfil lipídico: "los triglicéridos deben ser menores a 150 mg/dl”. Así pues, conviene subrayar que las desigualdades son parte importante en la relación y significación tanto de contextos matemáticos como de los cotidianos y para establecer relaciones entre estos dos. Es por esto que, en este trabajo presentamos una estrategia didáctica que tiene como objetivo fortalecer los procesos de comprensión y de significado del concepto de desigualdad al aplicarlo tanto en contextos matemáticos como en contextos cotidianos por medio del tratamiento y conversión de las representaciones semióticas. Para ello, se involucraron estudiantes de último año de bachillerato que desarrollaron la estrategia en tres fases didácticas del aprendizaje conceptual de las desigualdades. Primero, en la fase de las representaciones, se aplican tareas orientadas a correlacionar los diferentes sistemas de representación semiótica de las desigualdades; la segunda, en la fase de los tratamientos, se resuelven tareas para evaluar como los estudiantes solucionan inecuaciones lineales por medio los tratamientos semióticos y la tercera, para la fase de las conversiones, se construyeron y evaluaron tareas de conversión semiótica para interpretar contextos gráficos de dominios, codominios y perfiles lipídicos de una tabla de datos. El resultado de esta investigación, es proponer una estrategia didáctica que le permita al profesor identificar las fortalezas y debilidades del proceso de aprendizaje del concepto de desigualad a partir del significado que los estudiantes empelan al solucionar situaciones en multicontextos, aportando una herramienta educativa para aplicar en clase.

\section{MARCO TEÓRICO}

La generación del conocimiento matemático surge como consecuencia de la interacción entre el sujeto y los contextos problematizadores puesto que, el sujeto para intervenir a dichos contextos, debe transformar su representación mental del problema en diferentes representaciones semióticas con el objetivo de exteriorizar el significado de dicho contexto o problema (Duval, 2006; Godino, 2016).

Así pues, las representaciones semióticas son estructuras con significado propio siguiendo reglas formales del lenguaje natural, del álgebra, de las gráficas y del simbolismo (Guerrero-Ortiz et al., 2016); es por esto, que las representaciones semióticas tienen papel importante en el desarrollo y trasformación de las representaciones mentales provenientes de los contextos cotidianos y matemáticos.

De acuerdo a lo anterior, propongo dos tipos de contextos problematizadores como punto de partida para el aprendizaje conceptual de las desigualdades por medio de los registros de representaciónsemiótica, estosson:loscontextos cotidianos y los contextos matemáticos. Para algunos contextos cotidianos, los sujetos deben emplear el concepto de desigualdad para dotar de significado diversas situaciones problémicas de su entorno a través de representaciones semióticas de tipo lingüístico y numérico, con el fin de definir intervalos de medición; por ejemplo, la expresión "El peso de Saúl debe estar entre los 65 y 67 kilogramos"; emplea la desigualdad y un patrón de medida para comunicar significativamente el rango peso ideal de Saúl. En algunos contextos matemáticos, el concepto de desigualdad utiliza diferentes tipos de representaciones semióticas para darle sentido y significado a expresiones matemáticas, como por ejemplo, "el dominio de es $(0, \infty)$ "; esto hace 
referencia al comportamiento numérico en el que se define la función en el intervalo abierto entre cero e infinito.

\subsection{Los contextos para el aprendizaje conceptual}

Para Vergnaud (1998) el aprendizaje conceptual de las matemáticas se genera por la interacción entre el concepto y sus representaciones semióticas. Sin embargo, como se trató anteriormente, el contexto provee de significado a esta interacción con lo cual proponemos incluir al contexto como el componente inicial que permite configurar el aprendizaje conceptual de las desigualdades por medio de una triada como se muestra en la Figura 1.

\section{Figura 1}

Triada para el aprendizaje conceptual de las desigualdades matemáticas

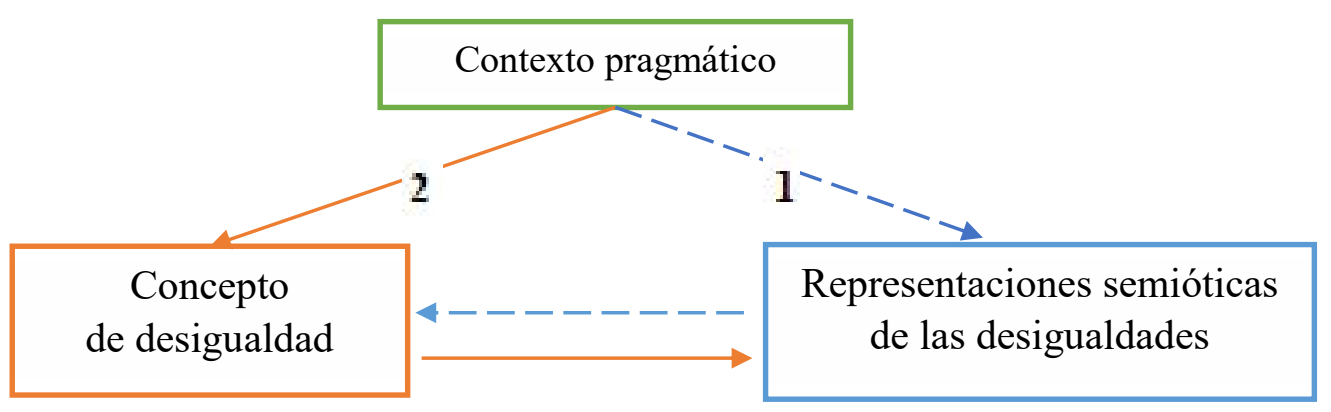

Fig. 1. Dos interpretaciones del contexto para el aprendizaje conceptual de las desigualdades

La triada de la figura 1 tiene su origen en el contexto. El contexto pragmático, como se explicó anteriormente, es un registro semiótico de tipo lingüístico que representa una idea matemática (contexto matemático) o una situación de la cotidianeidad (contexto cotidiano) en las que está inmersa el concepto de desigualdad. Así pues, el aprendizaje puede tener dos rutas didácticas para desarrollar los procesos de comprensión y significación conceptual; la ruta 1 (línea punteada): sigue la vía del contexto-representaciones semióticasconcepto de desigualdad; de esta manera el estudiante transforma el contexto lingüístico en uno o varios registros semióticos para luego llegar al concepto de desigualdad. La ruta 2 (línea continua): sigue el siguiente orden, contextoconcepto-representación semiótica, de esta manera, la estructura lingüística del contexto alude al concepto de desigualdad y luego se representa por múltiples registros semióticos

\subsection{Aprendizaje conceptual de las desigualdades}

Varios autores afirman (D'amore et al., 2013; Duval, 2017a; Fandiño, 2010; Vergnaud, 1998) que el aprendizaje conceptual depende de tres aspectos importantes (i) reconocer en el contexto el concepto de desigualdad y representarlo en determinados registros semióticos; (ii) efectuar tratamientos a las representaciones semióticas en un mismo registro semiótico de desigualdad y (iii) realizar conversiones entre registros de desigualdades de diferentes representaciones semióticas. A continuación, se explican en detalle cada uno de los tres aspectos para el caso del aprendizaje conceptual de las desigualdades.

\subsubsection{Representaciones semióticas del concepto de desigualdad}

Duval (2017b) afirma que los objetos matemáticos quedan invariantes una vez se 
definen, sin embargo, para poder acceder a los objetos matemáticos se requieren de múltiples registros de representación semiótica. Para el caso específico del objeto matemático de desigualdad, se logran identificar cuatro grupos de representaciones semióticas para acceder al concepto, estas son: lingüísticas, gráficas, notacionales y conjuntistas como se muestra en la Tabla 1. Estos cuatro grupos de representaciones semióticas de las desigualdades son la base para la construcción de estrategias didácticas que conlleven a analizar de los signos y el lenguaje que configura la actividad semiótica de las desigualdades como objeto matemático en cuestión (lori, 2016). En la tabla I se muestran los cuatro grupos de representaciones semióticas y la equivalencia entre sus registros, de esta manera se pueden establecer múltiples relaciones para representar el mismo concepto de desigualdad, ya sea en un contexto matemático o cotidiano.

\section{Tabla 1}

\section{Cuatro grupos de representaciones semióticas del concepto de desigualdad}

Representaciones semióticas del concepto de desigualdad

\begin{tabular}{ccccc}
$\begin{array}{c}\text { Representacion } \\
\text { es } \\
\text { Lingüísticas }\end{array}$ & $\begin{array}{c}\text { Representacione } \\
\text { Gráficas }\end{array}$ & $\begin{array}{c}\text { Representacione } \\
\text { Notación }\end{array}$ & \multicolumn{2}{c}{$\begin{array}{c}\text { Representaciones de } \\
\text { Conjunto }\end{array}$} \\
\hline Intervalo abierto & & $(a, b)$ & $A=\{x \in \mathbb{R} / a<x$ & $a<x<b$ \\
& & & & \\
Intervalo \\
cerrado
\end{tabular}

Las desigualdades están determinadas por sistemas simbólicos, gráficos y lingüísticos que hacen de ellas un campo enriquecedor para el aprendizaje conceptual. Así pues, las representaciones lingüísticas son de carácter verbal y escrito, y cuya utilidad es la de relacionar expresiones cotidianas con expresiones matemáticas y viceversa; esta relación dota de sentido y significado a cada uno de estas dos expresiones. Conceptualmente, la palabra "abierto" en el lenguaje de las desigualdades significa que no se incluyen valores los extremos de la desigualdad; mientras que, la palabra "cerrado" significa que se incluyen estos valores. De la misma manera, los términos "semiabiertos" o también llamados "semicerrados" incluyen o 
excluyen respectivamente los valores extremos "a izquierda o a derecha" de una misma desigualdad.

Las representaciones gráficas usualmente se empelan como punto de partida en las prácticas de aula puesto que, los estudiantes logran visualizar fácilmente a través de un gráfico (recta numérica) todos los posibles valores que comprenden la desigualdad. De esta manera, la inclusión o exclusión de los valores extremos de un intervalo en una recta numérica se representan gráficamente por dos tipos de punto ○ y •, con lo cual, el significado del punto "○" corresponde a la exclusión del valor límite y el punto "•" corresponde a la inclusión del valor límite de la desigualdad matemática.

Por otro lado, las representaciones notacionales se caracterizan por la utilización de signos de puntuación como "( )" y "[ ]"; en donde el paréntesis representa la exclusión del valor extremo de un intervalo, mientras que el corchete incluye el valor extremo del intervalo. Estas representaciones se usan frecuentemente para definir valores o condiciones numéricas en teoremas y definiciones propias de las matemáticas.
En cuanto a las representaciones conjuntistas, estas se fundamentan en los conceptos lingüísticos "mayor que", "menor que", "mayor o igual que" y "menor o igual que", que simbólicamente corresponden a y se utilizan para establecer los valores extremos de un intervalo definido en un conjunto numérico. Así pues, los registros semióticos que usan los símbolos "<" y ">" significan la exclusión de valores extremos, mientras que, los símbolos " $\leq$ " y "z" significa la inclusión de estos valores.

\subsubsection{El tratamiento de los registros de representación semiótica de las desigualdades}

El tratamiento semiótico se define como la transformación que se efectúa entre registros al interior de una misma representación (Duval, 2017a). El tratamiento semiótico se caracteriza por seguir reglas determinadas de funcionamiento y por generar tan solo un nuevo registro de representación (Duval, 2017b). Para el caso de las desigualdades, los tratamientos de los registros semióticos siguen las siguientes propiedades aritméticas (Tabla 2). De esta manera, la aplicación de una o varias de estas propiedades corresponde a uno o varios tratamientos.

Tabla 2

Propiedades aritméticas de las desigualdades

Sean $x, y$ and $z$ números reales

\begin{tabular}{ll}
\hline Propiedad de la adición & Si $x<y$ entonces $x+z<y+z$ \\
\hline Propiedad de la sustracción & Si $x<y$ entonces $x-z<y-z$ \\
\hline Propiedad de la multiplicación & Si $x<y$ and $z>0$ entonces $x z<y z$ \\
\hline Propiedad de la división & Si $x<y$ and $z<0$ entonces $x z>y z$ \\
\hline & Si $x<y$ and $z>0$ entonces $x / z<y / z$ \\
\hline
\end{tabular}

Propiedades necesarias para el tratamiento semiótico de las desigualdades 
En la tabla 2 se observa que, los tratamientos o las propiedades de suma y resta no alteran el sentido de la desigualdad; sin embargo, en las multiplicaciones y en las divisiones las desigualdades cambian de sentido cuando se operan por un número real negativo; este último caso, es relevante en el estudio del aprendizaje conceptual de las desigualdades puesto que, los estudiantes cometen errores con frecuencia al aplicar estas propiedades (Balomenou et al., 2017). Por tanto, la enseñanza y aprendizaje del tratamiento de los registros semióticos de las desigualdades debe darse con sumo cuidado para evitar errores en sus aplicaciones, así lo explica D'Amore (2004) se evidencia la principal dificultad para el aprendizaje de conceptos está en el tratamiento, ya que muchos estudiantes carecen de las herramientas o del dominio de las propiedades para relacionar y construir nuevos registros semióticos a partir del registro inicial

Ahora bien, las desigualdades son empleadas para modelar inecuaciones que explican diferentes fenómenos matemáticos (contextos matemáticos o cotidianos) y los tratamientos semióticos se aplican paso a paso para proveer la solución al modelado. Así pues, la cantidad de tratamientos semióticos necesarios para solucionar inecuaciones dependerá del número de veces que se apliquen las propiedades de tabla 2 hasta obtener el conjunto de soluciones numéricas requeridas.

\subsubsection{La conversión de los registros de representación semiótica de las desigualdades}

La conversión semiótica se define como la transformación que se efectúa entre registros de distintas representaciones (Duval, 2017b). Y se caracteriza por permitir pasar de un registro a otro de manera coordinada por el sujeto (Duval, 2017a). Esto significa que, la conversión semiótica de las desigualdades, consiste en coordinar múltiples transformaciones entre los registros de los cuatro grupos de representaciones semióticas caracterizados en la Tabla 1. (ver ejemplo Fig. 2)

Figura 2

Sistema integrado de conversiones semióticas para el aprendizaje conceptual de las desigualdades

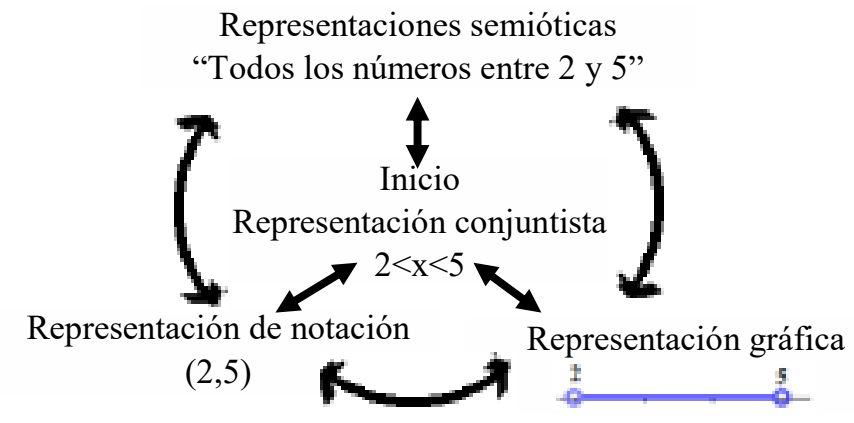

Fig. 2. Conversión de la representación inicial conjuntista en otras representaciones semióticas de las desigualdades 
La Fig. 2 muestra las posibles conversiones semióticas que tiene la representación inicial de conjunto " $2<x<5$ ", este registro puede pasar a un registro de representación gráfica, lingüística, de notación y en sentido opuesto; así mismo, se observa que, los registros de representaciones gráficas, lingüísticas y de notación tienen su propia relación sin conectar con el registro inicial. Esta coordinación entre registros equivalentes, permite seleccionar la representación más pertinente para interpretar contextos matemáticos o cotidianos. Pues, el objetivo de las conversiones de los registros semióticos es permitirle al sujeto (estudiante) la posibilidad de asociar múltiples representaciones a la semántica que define un problema de desigualdades con sentido y significado. Por tanto, la interpretación que haga el estudiante de un problema con desigualdades, dependerá del dominio conceptual y de la capacidad de asociar o modelar una inecuación inicial para ser tratada semióticamente hasta llegar a su solución numérica.

En resumen, el aprendizaje conceptual de las desigualdades se considera que está cognitivamente adquirido cuando el estudiante esta en grado de: identificar las propiedades del concepto en un determinado contexto y por tanto lo puede representar de manera adecuada, y si la situación lo exige deberá tratar y convertir semióticamente dicho contexto (Fandiño, 2010).

\section{METODOLOGÍA}

En esta investigación busca comprender, describir y analizar las interpretaciones que realizan los estudiantes en su proceso de aprendizaje del concepto de desigualdad con el objetivo de proponer una estrategia didáctica para el aprendizaje conceptual de las desigualdades con significado. Para esto, el diseño de la estrategia didáctica basado en las representaciones semióticas pretende potenciar los tres aspectos de las representaciones semióticas explicados en las secciones 2.2.1
- 2.2.3 y que apuntan a resolver problemas en contextos cotidianos. De esta manera se utiliza un enfoque cualitativo con el fin de realizar estudios profundos de fenómenos interpretativos basados justamente en las prácticas realizadas por los estudiantes (Hernandez et al., 2010).

Lainvestigaciónsedesarrollóenel primerbimestre del año escolar y contó con la participación de 37 estudiantes con edades comprendidas entre los 15 y 16 años. La estrategia didáctica para el aprendizaje conceptual de las desigualdades fue aplicada a dos cursos de precálculo de una institución educativa privada de la ciudad de Barrancabermeja (Colombia).

\subsection{Estructura de la estrategia didáctica para el aprendizaje conceptual de las desigualdades}

La estrategia didáctica se fundamenta en los procesos del aprendizaje conceptual de las representaciones, tratamiento y conversión de las desigualdades. Estos tres aspectos le permiten al profesor analizar las interpretaciones empleadas por los estudiantes al solucionar problemas tanto de contextos matemáticos como cotidianos. De ahí que, el diseño y construcción de la estrategia didáctica siga metodológicamente el orden de las tres fases (secciones 2.2.1 - 2.2.3) para el aprendizaje conceptual de las desigualdades por medio de tareas específicas, sistemáticas y orientadas al logro propuesto para cada fase del aprendizaje de las desigualdades (Fig.3). 


\section{Figura 3}

Estructura metodológica de la estrategia didáctica para el aprendizaje conceptual de las desigualdades

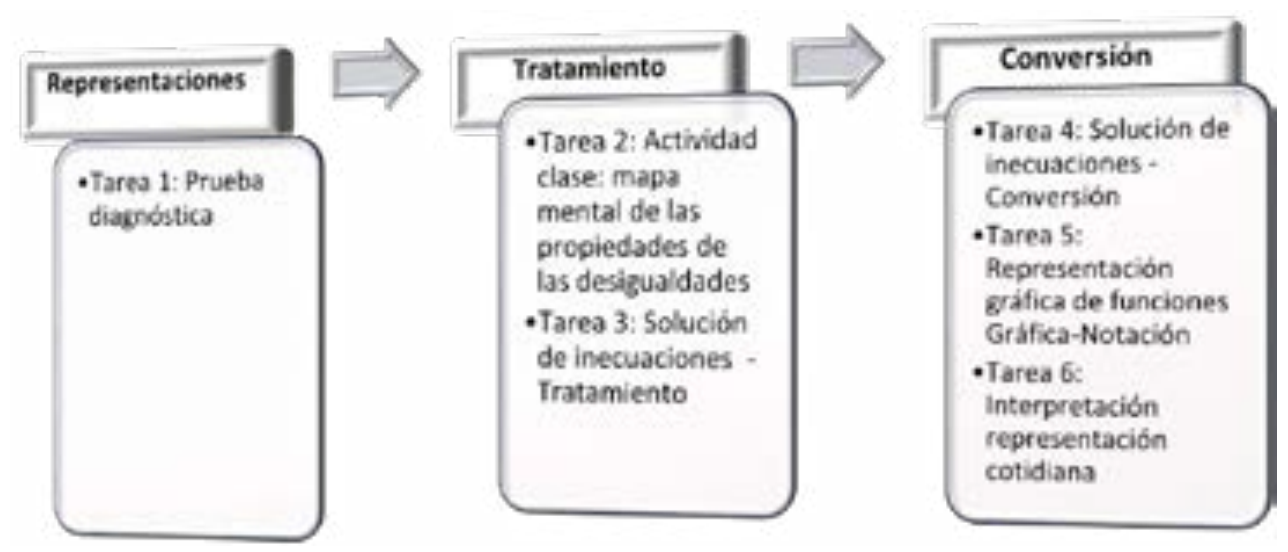

Fig. 3. Fases y contenidos de la estrategia didáctica para el aprendizaje conceptual de las desigualdades

\subsubsection{Primera fase: las representaciones semióticas de las desigualdades}

La tarea 1 es la prueba diagnóstica devela las ideas previas que posee los estudiantes sobre de las representaciones semióticas de las desigualdades relacionadas a la Tabla 1.

\subsubsection{Segundafase:Iostratamientos semióticos de las desigualdades}

Esta fase está diseñada para analizar cómo los estudiantes aplican las propiedades aritméticas de las desigualdades en la solución de inecuaciones lineales para determinar el intervalo solución. Para esto se proponen las tareas 2 y 3 ; en la tarea 2 los estudiantes crean autónomamente un mapa mental con las propiedades aritméticas de las desigualdades (Tabla 2) con el fin de reconocer los cambios que pueden o no tener las desigualdades al sumar, restar, multiplicar, dividir tanto con reales positivos como negativos. En la tarea 3, se plantean tres inecuaciones lineales que requieren de la aplicación de las propiedades aritméticas (Tabla 2) y su conversión gráfica (Tabla 1); esta tarea se diseñó para reconocer el dominio conceptual de las propiedades aritméticas de las desigualdades (Tabla 2) en cada uno de los tratamientos semióticos aplicados al resolver de inecuaciones, es por esto que, se proponen inecuaciones con diferentes niveles de dificultad, lo que permite identificar cómo los estudiantes pasan de tratamientos básicos a más complejos al solucionar problemas en contextos matemáticos

\subsubsection{Tercera fase: las conversiones semióticas de las desigualdades}

Finalmente, la tercera fase plantea las tareas 4,5,6 con el objetivo de convertir algunos contextos cotidianos y matemáticos en diversas representaciones semióticas para darle sentido y significado a la información presentada. De esta manera, la tarea 4 busca convertir los resultados numéricos de una inecuación lineal en múltiples representaciones semióticas equivalentes, específicamente en representaciones de conjunto, notación, gráfica y lingüística. El propósito de estas conversiones, es identificar cómo los estudiantes crean y sustituyen con sentido y significado el resultado 
numérico de una inecuación lineal en otra representación semiótica equivalente. La tarea 5 por su parte, le propone al estudiante interpretar el dominio y codominio de cada registro gráfico para determinar su registro simbólico de función. Finalmente, la estrategia didáctica termina con la tarea 6 orientada a interpretar datos de asuntos cotidianos. Para esto se propone la lectura de la tabla de valores de diferentes parámetros asociados al perfil lipídico de una persona. En este se establecen los valores mínimos y máximos de varios tipos de colesterol y de triglicéridos (representación lingüística cotidiana) cuyo objetivo es analizar las interpretaciones y conversiones que el estudiante realiza entre diferentes tipos de registros de representaciones semióticas.

En conclusión, el conjunto ordenado de tareas que conforman la estrategia didáctica y que fueron aplicadas clase, permitió indagar a través de las representaciones semióticas, el aprendizaje de las desigualdades para estudiantes de último año de secundaria. De ahí la importancia de la estrategia; puesto que, es una herramienta que permite realizar seguimiento sistematizado a los significados que emplea cada estudiante al solucionar problemas multicontextos propiciando información precisa y singular del proceso de aprendizaje.

\subsection{Recolección y análisis de la información}

La estrategia didáctica está compuesta por seis tareas (Fig. 3) que aportaron información precisa de cada estudiante en su proceso de aprendizaje conceptual de las desigualdades. Así pues, cada tarea fue diseñada con la intención de proveer información de los significados que emplean los estudiantes al interpretar, comprender y solucionar situaciones en contextos matemáticos y cotidianos, como se muestra en la Tabla 3:

Tabla 3

Instrumentos y análisis de resultados de la estrategia según las tres fases

\begin{tabular}{|c|c|c|c|}
\hline Fases de la estrategia & $\begin{array}{c}\mathrm{N}^{\circ} \text { de la } \\
\text { tarea }\end{array}$ & Instrumento & Análisis \\
\hline $\begin{array}{c}\text { Primera Fase } \\
\text { Representaciones } \\
\text { Semióticas }\end{array}$ & Tarea 1 & Prueba diagnóstica & $\begin{array}{l}\text { Presaberes de } \\
\text { los } 5 \text { tipos de } \\
\text { representaciones } \\
\text { semióticas de las } \\
\text { desigualdades }\end{array}$ \\
\hline Segunda Fase & Tarea 2 & Mapa mental & $\begin{array}{l}\text { Reconocimiento de las } \\
\text { propiedades aritméticas } \\
\text { de las desigualdades }\end{array}$ \\
\hline $\begin{array}{l}\text { Tratamientos } \\
\text { semióticos }\end{array}$ & Tarea 3 & $\begin{array}{l}\text { Inecuaciones } \\
\text { lineales }\end{array}$ & $\begin{array}{l}\text { Solución paso a paso } \\
\text { de tres inecuaciones } \\
\text { con diferente nivel de } \\
\text { dificultad }\end{array}$ \\
\hline
\end{tabular}




\begin{tabular}{clll}
\hline & Tarea 4 & $\begin{array}{l}\text { Conversión de } \\
\text { inecuaciones }\end{array}$ & $\begin{array}{l}\text { Transformar la } \\
\text { respuesta de una } \\
\text { inecuación a otros } \\
\text { registros semióticos } \\
\text { Tercera Fase }\end{array}$ \\
\cline { 2 - 4 } Conversiones & Tarea 5 & Conversión gráfica & $\begin{array}{l}\text { Transformación de la } \\
\text { gráfica de una función } \\
\text { a representaciones } \\
\text { simbólicas y de } \\
\text { desigualdad }\end{array}$ \\
\cline { 2 - 4 } & & Perfil lipídico & $\begin{array}{l}\text { Transformación de } \\
\text { representaciones } \\
\text { semánticas y numéricas } \\
\text { a desigualdades }\end{array}$ \\
\hline
\end{tabular}

Estructura de la estrategia didáctica por fases, tareas, instrumentos y acciones para el aprendizaje de las desigualdades

La Tabla 3 muestra ordenadamente explícito de la relación entre las fases, las tareas, los instrumentos y acciones tal como fueron aplicadas en clase para el aprendizaje conceptual de las desigualdades.

\section{RESULTADOS}

Como resultado de esta investigación, se muestra en detalle la estrategia didáctica que fue diseñada, creada y aplicada en el orden metodológico propuesto para el aprendizaje conceptual. El orden metodológico divide la estrategia en las tres fases mostradas en la figura 3, que siguen las ideas expresadas por D'amore et al. (2013); Duval (2017a); Fandiño (2010); Vergnaud (1998); además, fueron evaluadas al finalizar cada una de ellas. De ahí que, para cada una de las fases, se definen una serie de tareas para desarrollar en clase con estudiantes, las cuales sintetiza los aspectos más relevantes del aprendizaje conceptual de las desigualdades, teniendo como referente el análisis de las interpretaciones y los procedimientos realizados por los participantes en varios temas de interés, donde el concepto de desigualdad desempeña un papel crucial. Estos temas están relacionados con la solución de problemas: i) de inecuaciones, ii) en contextos matemáticos y iii) en contextos cotidianos.

\subsection{Primera fase: las representaciones semióticas de las desigualdades}

Esta fase se desarrolla con la tarea 1 que se describen a continuación:

Objetivo de la fase: reconocer y afianzar los conocimientos preexistentes de los estudiantes sobre las representaciones semióticas de las desigualdades por medio de una prueba diagnóstica y un mapa conceptual.

Objetivo de la tarea 1: evaluar los preconceptos de las representaciones semióticas de las desigualdades que poseen los estudiantes caracterizados en la Tabla 1. 


\section{Figura 4}

Tarea 1: prueba diagnóstica para la estrategia didáctica para el aprendizaje de las desigualdades Complete la siguiente tabla con las representaciones que corresponda:

\begin{tabular}{|c|c|c|c|c|}
\hline $\begin{array}{c}\text { Representación } \\
\text { Lingüística } \\
\text { (nombre) }\end{array}$ & Gráfica & Notación & Conjunto & Lingüística \\
\hline Abierto & & & & (Interpretación) \\
\hline Cerrado & & {$[2,5]$} & & \\
\hline Semiabierto & & & & \\
\hline
\end{tabular}

Fig 4. Prueba diagnóstica de preconceptos de las representaciones semióticas de las desigualdades

Desarrollo de la actividad: los estudiantes completan la tabla de Prueba Diagnóstica al convertir cada registro semiótico inicial en otros registros semióticos equivalentes; por ejemplo, los estudiantes convierten la representación gráfica de abierto, propuesta en la tabla, en representación de notación $(-2,3)$, en representación de conjunto $-2<x<3$ y en representaciones lingüísticas de interpretación: "todos los valores numéricos que están entre -2 y 3 ", en esta última, los estudiantes expresan por escrito el significado que le da a la representación. La interconexión de las conversiones semióticas le permite al profesor identificar la capacidad que tiene cada estudiante para relacionar el significado de desigualdad con cada representación semiótica.

\subsection{Segunda fase: el tratamiento semiótico de las desigualdades}

Esta fase está constituida por las tareas 2 y 3 , como se muestran a continuación:

Objetivo de la fase: caracterizar y aplicar las propiedades aritméticas de las desigualdades en la solución de inecuaciones lineales para representarlas gráficamente y simbólicamente.
Objetivo de la tarea 2: diseñar un mapa mental de las propiedades aritméticas de las desigualdades junto con su representación gráfica.

Desarrollo de la actividad: cada estudiante autónomamente crea un mapa mental para reconocer los cambios en los registros numéricos, gráficos y de signo que tiene la desigualdad al realizar sumas, restas, multiplicaciones y divisiones con números reales descritos en la Tabla 2.

Objetivo de la tarea 3: aplicar tratamientos aritméticos (Tabla 2) para resolver inecuaciones con diferentes niveles de dificultad. 


\section{Figura 5}

Tarea 3: solución de inecuaciones lineales

Aplique las propiedades de las desigualdades para resolver paso a paso las siguientes inecuaciones:

$\begin{array}{ll}\text { a) } 5 x-4<3 x+5 & \text { b) } \frac{x}{5}+\frac{x}{3}-1<\frac{x}{2}\end{array}$ c) $\frac{x-5}{3}+\frac{x+4}{2} \geq \frac{x+3}{6}$

Fig. 5 Fase del tratamiento semiótico: aplicación de las propiedades de las desigualdades para solucionar inecuaciones lineales.

Desarrollo de la actividad: se presentan tres inecuaciones para que los estudiantes apliquen paso a paso los tratamientos semióticos fundamentados en las propiedades aritméticas (tabla 2) hasta llegar a la respuesta. Cada inecuación difiere de las otras dos por su nivel de dificultad; así pues, la inecuación a) es básica al resolver puesto que, los tratamientos se basan en las propiedades de suma y resta de números enteros $(\mathbb{Z})$ y agrupación de variables; la inecuación b) exige tratamientos numéricos y algebraicos con racionales, por lo que se necesita del dominio de aritmético de los números fraccionaros aumentando la cantidad y dificultad de tratamientos con respecto a la inecuación a); de manera similar la inecuación c), exige del dominio numérico racional pero, los tratamientos algebraicos son preponderantes para solucionar la inecuación correctamente, maximizando así la complejidad de la inecuación.

Al finalizar esta fase, los estudiantes deben haber conseguido relacionar los cuatro tipos de representaciones semióticas de las desigualdades con las propiedades aritméticas que se emplean en los tratamientos para solucionar inecuaciones con el objetivo de proporcionarle significado al concepto de desigualdad en dos estadios diferentes.

\subsection{Tercera fase: las conversiones semióticas de las desigualdades}

Esta fase contiene las tareas $4,5,6$ como se muestran a continuación:

Objetivo de la fase: convertir cada representación semiótica inicial de una desigualdad en múltiples registros semióticos equivalentes para resolver problemas tanto de contextos matemáticos como cotidianos

Objetivo de la tarea 4: aplicar tratamientos aritméticos a una inecuación (Tabla 2) para calcular su solución, representarla gráficamente y simbólicamente (Tabla 1).

\section{Figura 6}

Tarea 4: solución de inecuaciones y conversión semiótica del resultado

Resuelva la inecuación aplicando las propiedades de las desigualdades (tratamientos) y convierta su resultado en otras representaciones semióticas en cada caso:

\begin{tabular}{|c|l|l|l|l|}
\hline Inecuación & $\begin{array}{l}\text { representación } \\
\text { de conjunto }\end{array}$ & $\begin{array}{l}\text { representación } \\
\text { de notación }\end{array}$ & $\begin{array}{l}\text { representación } \\
\text { gráfica }\end{array}$ & $\begin{array}{l}\text { representación } \\
\text { lingüística }\end{array}$ \\
\hline$\frac{4 x+1}{3} \leq \frac{12 x-3}{7}$ & & & & \\
\hline
\end{tabular}

Fig 6. Conversiones semióticas del resultado de la inecuación a otros tipos de representaciones 
Desarrollo de la actividad: para resolver la inecuación propuesta, los estudiantes deben aplicar tratamientos semióticos de suma, resta y multiplicación con números positivos, negativos y fraccionarios (Tabla 2) haciendo hincapié en la propiedad de multiplicar por un negativo puesto que, en el proceso de solución es necesario cambiar la dirección de la desigualdad para obtener un resultado correcto. Después de los tratamientos, los estudiantes deben convertir la respuesta a representaciones de notación, gráficas y lingüísticas; de esta forma se comprueba que el estudiante ha adquirido solidez en la significación del concepto de desigualdad.

Objetivo de la tarea 5: Definir y escribir el dominio, codominio y simbólicamente la función que corresponde a la representación gráfica.

Figura 7

Tarea 5: representación gráfica de funciones a representación de notación Escriba la función de las siguientes gráficas junto a su dominio y codominio.

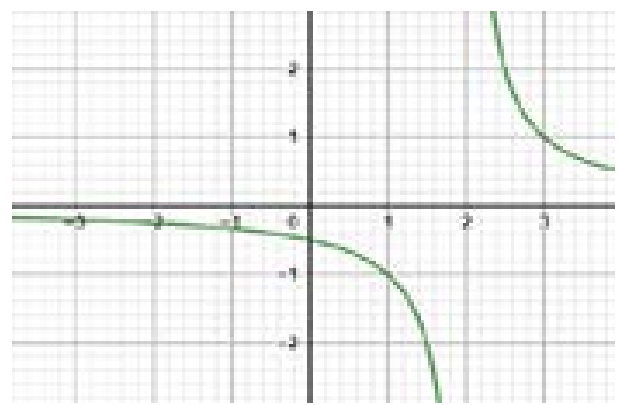

Función:

Dominio:

Codominio:

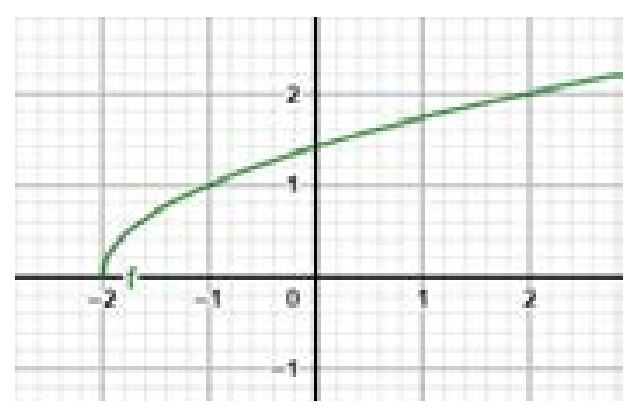

Función:

Dominio:

Codominio:

Fig. 7 Tareas para evaluar la conversión entre representaciones simbólicas, gráficas y de desigualdad

Desarrollo de la actividad: a partir del entorno gráfico de una función racional y otra función irracional, los estudiantes escriben matemáticamente la función " $f(x)=$ " que ellos interpretan según las características de la forma limitada por las asíntotas y que precisa el dominio y codominio de dicha representación gráfica. La relación entre la representación gráfica, la representación simbólica y las representaciones de las desigualdades crean un sistema integrado de significación del concepto de desigualdad que puede ser aplicado a otros contextos matemáticos.
Objetivo de la tarea 6: interpretar los registros numéricos de un perfil lipídico para convertirlos en representaciones semióticos de desigualdades matemáticas 


\section{Figura 8}

Tarea 6: Interpretación semántica y numérica de un perfil lipídico

En la siguiente tabla se muestran datos de los niveles normales de colesterol y triglicéridos:

\begin{tabular}{|l|c|c|c|l|}
\hline \multirow{2}{*}{ Parámetro } & \multicolumn{4}{|c|}{ Valores Normales o Valores de Referencia } \\
\cline { 2 - 5 } & Óptimo & $\begin{array}{c}\text { Sobre el límite } \\
\text { óptimo }\end{array}$ & Alto & Muy alto \\
\hline HDL-Colesterol & $\begin{array}{c}\text { Entre } 40 \text { y } 60 \mathrm{mg} / \\
\mathrm{dL}\end{array}$ & \multicolumn{2}{|c|}{ Si supera los $60 \mathrm{mg} / \mathrm{dL}$ es beneficioso } \\
\hline LDL-Colesterol & $\begin{array}{c}\text { Menor a } 100 \mathrm{mg} / \\
\mathrm{dL}\end{array}$ & $\begin{array}{c}\text { Entre } 100-129 \mathrm{mg} / \\
\mathrm{dL}\end{array}$ & $\begin{array}{c}\text { Entre 130-189 mg/ } \\
\mathrm{dL}\end{array}$ & $\begin{array}{l}\text { Mayor a } 190 \\
\mathrm{mg} / \mathrm{d}\end{array}$ \\
\hline Colesterol total & $\begin{array}{c}\text { Menor a } 200 \mathrm{mg} / \\
\mathrm{dL}\end{array}$ & \multicolumn{2}{|c|}{ Entre $200 \mathrm{mg} / \mathrm{dL}$ y $240 \mathrm{mg} / \mathrm{dL}$} & $\begin{array}{l}\text { Mayor a } 240 \\
\mathrm{mg} / \mathrm{d}\end{array}$ \\
\hline TRIGLICÉRIDOS & $\begin{array}{c}\text { Menor a } 150 \mathrm{mg} / \\
\mathrm{dL}\end{array}$ & $\begin{array}{l}\text { Entre } 150-199 \mathrm{mg} / \\
\mathrm{dL}\end{array}$ & $\begin{array}{c}\text { Entre 200-499 mg/ } \\
\mathrm{dL}\end{array}$ & $\begin{array}{l}\text { Mayor a } 500 \\
\mathrm{mg} / \mathrm{d}\end{array}$ \\
\hline
\end{tabular}

Escriba las representaciones de los siguientes datos:

\begin{tabular}{|l|l|}
\hline HDL- & Conjunto: \\
Colesterol: & \\
Alto & \\
\hline $\begin{array}{l}\text { Colesterol } \\
\text { Total: Muy alto }\end{array}$ & Lingüístico: \\
\hline
\end{tabular}

\begin{tabular}{|c|c|}
\hline $\begin{array}{l}\text { Triglicéridos: } \\
\text { óptimo }\end{array}$ & \multicolumn{1}{|c|}{ Notación: } \\
\hline $\begin{array}{l}\text { LDL-Límite } \\
\text { óptimo }\end{array}$ & Gráfico: \\
\hline
\end{tabular}

Fig. 8 conversión de datos del perfil lipídico a representación equivalentes de desigualdades.

Desarrollo de la actividad: la tabla muestra representaciones semánticas y numéricas que definen los valores normales de un perfil lipídico. Los estudiantes deben convertir diferentes representaciones semánticas a otro tipo de representaciones como de conjunto, de notación, gráficas y lingüísticas, demostrando dominio conceptual de las desigualdades aplicadas a situaciones cotidianas. Así pues, adquirida la estructura conceptual de las desigualdades pueden ser aplicadas a multicontextos ya sean para interpretar y comprender fenómenos matemáticos o cotidianos.

Para finalizar, es pertinente poner en conocimiento el tiempo que tomó la aplicación de la estrategia didáctica para el aprendizaje conceptual de las desigualdades. El tiempo corresponde a un periodo académico que consta de 10 semanas por 5 horas semanales; así mismo, se destaca que durante el desarrollo de las prácticas de la estrategia se resolvían preguntas individuales y de grupo; sin embargo, todo el desarrollo de la estrategia fue de carácter individual para identificar los progresos, retrasos y tiempos de aprendizaje de cada estudiante participante.

\section{CONCLUSIONES}

Los estudiantes que dominan las operaciones entre fracciones obtienen buen desempeño con los tratamientos semióticos de las desigualdades y calculan de manera efectiva la respuesta numérica. Esto comprueba que, el resultado numérico de la inecuación no sólo depende de la aplicación de las propiedades de las desigualdades, sino también, depende de 
los tratamientos a los sistemas numéricos en los que esté escrita la desigualdad.

Crear diferentes alternativas de conversión semiótica de las desigualdades dinamiza el conocimiento matemático desde la perspectiva matemática y la cotidiana. Prueba de ello, fue comprender matemáticamente la tabla de colesterol y triglicéridos pues, los estudiantes ya habían escuchado hablar de ello y eso despertó el interés de aprender.

Los procesos de significación del concepto de desigualdad se fortalecían a medida que avanzaba la estrategia didáctica puesto que, el aprendizaje se consolidaba lentamente desde el entretejido entre las representaciones semióticas, pasando por los tratamientos hasta las conversiones de las desigualdades en diferentes contextos.

\section{REFERENCIAS BIBLIOGRÁFICAS}

Almog, N., \& Ilany, B. S. (2012). Absolute value inequalities: High school students' solutions and misconceptions. Educational Studies in Mathematics, 81(3), 347-364. https:// doi.org/10.1007/s10649-012-9404-z

Balomenou, A., Komis, V., \& Zacharos, K. (2017). Handling Signs in Inequalities by Exploiting Multiple Dynamic Representations - the Case of ALNuSet. Digital Experiences in Mathematics Education, 3(1), 39-69. https://doi.org/10.1007/s40751-017-00299

Blanco, L. J., \& Garrote, M. (2007). Difficulties in learning inequalities in students of the first year of pre-university education in Spain. Eurasia Journal of Mathematics, Science and Technology Education, 3(3), 221-229. https://doi.org/10.12973/ejmste/75401
D'Amore, B. (2003). The noetic in mathematics. Scientia Pedagogica Experimentalis, $X X X I X, \quad 75-82 . \quad$ http://www.dm.unibo.it/ rsddm/it/articoli/damore/462 The noetic in math.pdf

D’Amore, B. (2004). Conceptualización, registros de representaciones semióticas y noética. Uno. Revista de Didáctica de Las Matemáticas., 35, 90-106.

D’amore, B., Fandiño, M., \& Lori, M. (2013). La semiótica en la didactica de la matemática. 181.

D’Amore, B., Fandiño, M., Marazzani, I., \& Sbaragli, S. (2010). La Didáctica y la Dificultad En Matemática (Primera Ed). Editorial Magisterio.

Duval, R. (2006). A cognitive analysis of problems of comprehension in a learning of mathematics. Educational Studies in Mathematics, 61(1-2), 103-131. https:// doi.org/10.1007/s10649-006-0400-z

Duval, R. (2017a). Semiosis Y Pensamiento Humano (Segunda Ed). Editorial Universidad del Valle.

Duval, R. (2017b). Understanding the Mathematical Way of Thinking - The Registers of Semiotic Representations. Springer Nature.

Fandiño, M. (2010). Multiples aspectos del aprendizaje de la matemática: evaluar e intervenir en forma mirada y especifica. Editorial Magisterio.

Geary, D., Berch, D., Ochsendorf, R., \& Mann, K. (2017). 2017-Acquisition of Complex Arithmetic Skills and Higher-Order Mathematics Concepts (N. Levy (ed.)). Thomson Digital. 
Godino, J. D. (2016). Mathematical concepts , Their meanings and Understanding. Proceedings of $X X$ Conference of the International Group for the Psychology of Mathematics Education, 2, 417-425. https://www.ugr.es/ jgodino/articulos_ ingles/meaning_understanding.pdf

Guerrero-Ortiz, C., Mejía-Velasco, H. R., \& Camacho-Machín, M. (2016). Representations of a mathematical model as a means of analysing growth phenomena. Journal of Mathematical Behavior, 42, 109-126. https://doi. org/10.1016/j.jmathb.2016.03.001

Hattikudur, S., \& Alibali, M. W. (2010). Learning about the equal sign: Does comparing with inequality symbols help? Journal of Experimental Child Psychology, 107(1), 15-30. https://doi.org/10.1016/j. jecp.2010.03.004

Hernandez, R., Fernandez, C., \& Baptista, M. (2010). Metodología de la investigación (Quinta Edi). McGraw Hill.

Iori, M. (2016). Objects , signs, and representations in the semio-cognitive analysis of the processes involved in teaching and learning mathematics: A Duvalian perspective. Educational Studies in Mathematics. https://doi.org/10.1007/ s10649-016-9726-3

Sangwin, C. J. (2015). Inequalities, assessment and computer algebra. International Journal of Mathematical Education in Science and Technology, 46(1), 76-93. https://doi.org/1 0.1080/0020739X.2014.941424

Sierpinska, A., Bobos, G., \& Pruncut, A. (2011). Teaching absolute value inequalities to mature students. Educational Studies in Mathematics, 78(3), 275-305. https://doi. org/10.1007/s10649-011-9325-2

Tsamir, P., \& Almog, N. (2001). Students' strategies and difficulties: The case of algebraic inequalities. International Journal of Mathematical Education in Science and Technology, 32(4), 513-524. https://doi. org/10.1080/00207390110038277

Tsamir, Pessia, \& Bazzini, L. (2004). Consistencies and inconsistencies in students' solutions to algebraic 'singlevalue' inequalities. International Journal of Mathematical Education in Science and Technology, 35(6), 793-812. https://doi.or $\mathrm{g} / 10.1080 / 00207390412331271357$

Vergnaud, G. (1998). A comprehensive theory of representation for mathematics education. Journal of Mathematical Behavior, 17(2), $167-181$. 